\title{
The Square Kilometre Array: a physics machine for the 21st Century (Presentation Video)
}

\section{Philip Diamond}

Philip Diamond, "The Square Kilometre Array: a physics machine for the 21st Century (Presentation Video)," Proc. SPIE 9143, Space Telescopes and Instrumentation 2014: Optical, Infrared, and Millimeter Wave, 91430M (22 June 2014); doi: 10.1117/12.2063456

Event: SPIE Astronomical Telescopes + Instrumentation, 2014, Montréal, Quebec, Canada 


\title{
The Square Kilometre Array: a physics machine for the 21st Century (Presentation Video)
}

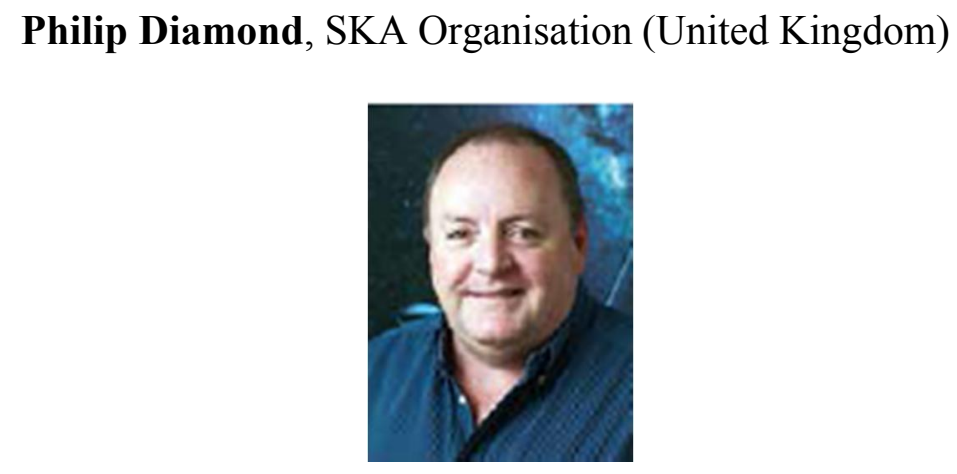

\begin{abstract}
The Square Kilometre Array is the next-generation radio-telescope. It will be a true mega-science facility. It is being designed and will be built by a global consortium, headquartered in the UK. The consortium currently has 11 member countries but is open for additional members at any time. The SKA Observatory will have sites in Australia and South Africa, and will build on the two precursor telescopes, ASKAP and MeerKAT, currently under construction on the sites. The SKA is being designed as a physics machine for the 21 st Century and will address scientific questions such as the nature of gravity, the origins of the Universe and the origins of life. I will describe the scientific rationale for the SKA; the technologies selected to deliver that science and the challenges posed in handling the massive data volumes to be generated by the observatory. The SKA is now in the detailed design phase. Funding exceeding $€ 120 \mathrm{M}$ has been committed by the partner nations to deliver that design. The design will be complete at the end of 2016 and, assuming construction funding is secured, the procurement process will begin in 2017 and construction in early 2018. The SKA will deliver early science by 2020 .
\end{abstract}

View presentation video on SPIE's Digital Library: http://dx.doi.org/10.1117/12.2063456.3664748679001 